\title{
NONEXISTENCE OF BEST ALTERNATING APPROXIMATIONS ON SUBSETS
}

\author{
CHARLES B. DUNHAM
}

\begin{abstract}
The existence of best Chebyshev approximations by an alternating family on closed subsets of an interval is considered. In varisolvent approximation, existence on subsets of sufficiently low density is guaranteed if the best approximation on the interval is of maximum degree. The paper studies the case in which the best approximation is not of maximum degree and shows that in many common cases, no such guarantee is possible.
\end{abstract}

Let $Y$ be a compact subset of $[\alpha, \beta]$ and $C(Y)$ be the space of continuous functions on $Y$. For $g \in C(Y)$ define

$$
\|g\|_{Y}=\sup \{|g(x)|: x \in Y\}, \quad\|g\|=\|g\|_{[\alpha, \beta]} .
$$

Let $F$ be an approximating function on $[\alpha, \beta]$ with parameter space $P$. The Chebyshev problem on $Y$ is, given $f \in C(Y)$, to find a parameter $A^{*}$ to minimize $\|f-F(A, \cdot)\|_{Y}$ over $A \in P$. Such a parameter $A^{*}$ is called best and $F\left(A^{*}, \cdot\right)$ is called best to $f$ on $Y$. We will consider only approximating functions for which best approximations on $[\alpha, \beta]$ are characterized by alternation [10, pp. 17-21], [6], which implies uniqueness of best approximations.

Following Cheney, we define the density of $Y$ in $[\alpha, \beta]$ to be

$$
|Y|=\max \{\inf \{|x-y|: y \in Y\}: \alpha \leqslant x \leqslant \beta\} .
$$

The best known alternating approximation functions are varisolvent approximating functions, discussed in Rice $[10,3 \mathrm{ff}]$ (we assume that the difficulty with a nonzero constant error curve [1] does not occur). In [7] it is shown that if $F$ is varisolvent and the best approximation to $f$ on $[\alpha, \beta]$ is of maximum degree, best approximations exist to $f$ on all subsets $Y$ of sufficiently low density. We wish to consider the case in which the best approximation on $[\alpha, \beta]$ is not of maximum degree.

1. Irregularity. The author's study of several alternating approximating functions suggests that a key property is that of irregularity, introduced in [4].

Definition. $F$ is $\alpha$-irregular at $A$ if for any triple $(x, y, \varepsilon), \alpha<x<\beta, y$ a real number, and $\varepsilon>0$, there is a parameter $B \in P$ such that $|F(B, \alpha)-y|$ $<\varepsilon$ and

Received by the editors October 2, 1975.

AMS (MOS) subject classifications (1970). Primary 41 A50. 


$$
|F(B, w)-F(A, w)|<\varepsilon, \quad x \leqslant w \leqslant \beta .
$$

Definition. $F$ is $\beta$-irregular at $A$ if for any triple $(x, y, \varepsilon), \alpha<x<\beta, y$ a real number, and $\varepsilon>0$, there is a parameter $B \in P$ such that $|F(B, \beta)-y|$ $<\varepsilon$ and

$$
|F(B, w)-F(A, w)|<\varepsilon, \quad \alpha \leqslant w \leqslant x .
$$

Not all alternating approximating functions of variable degree exhibit irregularity (an example of variable degree without irregularity is given in [6, pp. 104-105]) but all the "naturally occurring" ones seem to have this property.

2. Examples. It is shown in [4] that degenerate rationals and degenerate exponentials on $[0,1]$ are 0 -irregular. Similar arguments show that degenerate rationals on $[\alpha, \beta]$ are $\alpha$-irregular and $\beta$-irregular.

Let $F$ be an approximating function on $[\alpha, \beta], \alpha \geqslant 0$, such that $F(A, \cdot)$ being of less than maximum degree implies that $F(A, x)+\gamma \log (1+\delta x), \delta>-1 / \beta$, is an approximation. Such approximating functions are given in [3], [9]. Let $\delta_{k} \rightarrow-1 / \beta$ from above; then there is $\gamma_{k}$ such that $\gamma_{k} \log \left(1+\delta_{k} \beta\right)=y$ $-F(A, \beta)$. The arguments of [3, middle of p. 370] show that $\gamma_{k} \log \left(1+\delta_{k} w\right)$ $\rightarrow 0$ uniformly on $[0, x]$ for any fixed $x$ in $[0, \beta)$. Hence elements of less than maximum degree are $\beta$-irregular.

Let $F$ be an approximating function on $[0, \beta]$ such that $F(A, \cdot)$ being of less than maximal degree implies that $F(A, x)+\eta+\gamma \log (1+\sigma x), \sigma>-1 / \beta$, or $F(A, x)+\gamma \exp (\sigma x)$ is an approximation. A similar argument shows that $F$ is 0 -irregular in the first case and 0 -irregular and $\beta$-irregular in the exponential case.

\section{Notation.}

Definition. Let $\left\{X_{k}\right\}$ be a sequence of subsets of $[\alpha, \beta]$. We say $\left\{X_{k}\right\} \rightarrow[\alpha, \beta]$ if for any $x \in[\alpha, \beta]$, there is a sequence $\left\{x_{k}\right\} \rightarrow x, x_{k} \in X_{k}$. Let \|\|$_{k}$ denote the norm on $X_{k}$.

A point is called an extremum of $f-F\left(A^{*}, \cdot\right)$ if plus or minus the norm of $f-F\left(A^{*}, \cdot\right)$ is attained there. If each extremum is part of a (nondegenerate) closed interval of extrema, every sufficiently dense subset will contain alternating extrema of $f-F\left(A^{*}, \cdot\right)$ and $F\left(A^{*}, \cdot\right)$ will be best on the subset. That is why we require in the following theorem that the set of extrema be nowhere dense.

\section{Endpoint extrema.}

THEOREM 1. Let $F$ be $\alpha$-irregular at $A^{*}$. Let the set of extrema of $f-F\left(A^{*}, \cdot\right)$ be nowhere dense and $\alpha$ be an extremum. Then there exists a sequence $\left\{X_{k}\right\} \rightarrow[\alpha, \beta]$ such that no best approximation exists to $f$ on $X_{k}$.

Proof. Assume $f(\alpha)-F\left(A^{*}, \alpha\right)>0$. Let $e=f(\alpha)-F\left(A^{*}, \alpha\right), X_{k}=\{\alpha\}$ $\cup\left\{x:\left|f(x)-F\left(A^{*}, x\right)\right| \leqslant e-1 / k\right\}, 1 / k<e$.

By definition of irregularity, there is a sequence $F\left(A^{j}, \cdot\right)$ such that $\left|F\left(A^{j}, \alpha\right)-f(\alpha)\right|<1 / j$ and $F\left(A^{j}, \cdot\right) \rightarrow F\left(A^{*}, \cdot\right)$ uniformly on $X_{k} \sim\{\alpha\}$; hence $\left\|f-F\left(A^{j}, \cdot\right)\right\|_{k} \rightarrow e-1 / k$. Let $F\left(A^{*}, \cdot\right)$ have degree $l$; then there exists $x_{1}^{k}<\cdots<x_{l}^{k} \in X_{k}$ on which $f-F\left(A^{*}, \cdot\right)$ attains alternately $-e+1 / k$ and $e-1 / k$. Let $x_{0}=\alpha$. By the generalized lemma of de la Vallée-Poussin [2, p. 
226], we have for all $A \in P, F(A, \cdot) \not \equiv F\left(A^{*}, \cdot\right)$,

$$
\begin{aligned}
& \max \left\{\left|f\left(x_{i}^{k}\right)-F\left(A, x_{i}^{k}\right)\right|: i=0, \ldots, l\right\} \\
& \quad>\min \left\{\left|f\left(x_{i}^{k}\right)-F\left(A^{*}, x_{i}^{k}\right)\right|: i=0, \ldots, l\right\}=e-1 / k .
\end{aligned}
$$

As $f(\alpha)-F\left(A^{*}, \alpha\right)=e$, there is no approximant $F(A, \cdot)$ with $\|f-F(A, \cdot)\|_{k}$ $=e-1 / k$, that is, no best approximation to $f$ exists on $X_{k}$.

The same theorem holds for $\beta$-irregularity and $\beta$ an extremum.

The set $X_{k}$ in the proof of the theorem is infinite. If we want a finite set on which existence fails, we can choose $X_{k}^{\prime}$ a finite subset of $X_{k}$ such that

(i) $\left\{\alpha, x_{1}^{k}, \ldots, x_{l}^{k}\right\} \subset X_{k}^{\prime}$,

(ii) $X_{k-1}^{\prime} \subset X_{k}^{\prime}$,

(iii) dist $\left(X_{k}^{\prime}, X_{k}\right)<1 / k$.

5. Endpoints not extrema. If endpoints are not extrema, best approximations may exist on all subsets of sufficiently low density. Consider approximation by the family of ordinary rational functions $R_{1}^{0}[\alpha, \beta]$ of ratios $p / q$ of constants $p$ to first degree polynomials $q, q(x)>0$ for $\alpha \leqslant x \leqslant \beta$.

THEOREM 2. Let $R\left(A^{*}, \cdot\right)$ be best to $f$ in $R_{1}^{0}[\alpha, \beta]$ and no endpoint be an extremum. There exists a best approximation to $f$ on all subsets of sufficiently low density which contain the endpoints.

Proof. The only element of $R_{1}^{0}[\alpha, \beta]$ of less than maximum degree is the zero function, so we need only consider the case $R\left(A^{*}, \cdot\right)=0$. Choose $X$ such that it contains a point $x$ with

$$
|f(x)|>\max \{|f(\alpha)|,|f(\beta)|\} .
$$

Suppose no best approximation exists on $X$. A best generalized rational in the sense of Goldstein [10, pp. 85-88] exists and since it is not an ordinary rational, it must be of the form $0 /(a+b x), a+b x \geqslant 0$ for $x \in X$. It is, therefore, zero except on one endpoint. But from (1) it is easily seen that such a function is no better than zero and we have a contradiction.

Exactly the same theorem holds for approximation by

$$
F(A, x)=a_{1} \exp \left(a_{2} x\right)
$$

with subsets finite. The proof is the same except that in place of Goldstein's approach, we use the result that best approximations exist on finite $X$ if we add all functions zero except on an endpoint of $X$ [5].

6. Rationals with degeneracy $\geqslant 2$. Consider approximation by ordinary rationals $R_{m}^{n}[\alpha, \beta]$.

THEOREM 3. Let $R\left(A^{*}, \cdot\right)$ be the best approximation to $f$ on $[\alpha, \beta]$ by $R_{m}^{n}[\alpha, \beta]$ and have degeneracy two or more. Let the extrema of $f-R\left(A^{*}, \cdot\right)$ be nowhere dense. Then there is a sequence $\left\{X_{k}\right\} \rightarrow[\alpha, \beta]$ such that $f$ has no best approximation on $X_{k}$.

Proof. In view of Theorem 1 , we can assume that $f-R\left(A^{*}, \cdot\right)$ has an interior extremum $\gamma$ and that $f(\gamma)-R\left(A^{*}, \gamma\right)>0$. Let $e=\left\|f-R\left(A^{*}, \cdot\right)\right\|, X_{k}$ 
$=\gamma \cup\left\{x:\left|f(x)-R\left(A^{*}, x\right)\right| \leqslant e-1 / k\right\}, 1 / k<e$. Let $R\left(A^{*}, \cdot\right)=p / q, p$ of degree $n-2, q$ of degree $m-2, q(\gamma)=1$. Define

$$
R\left(A^{j}, x\right)=\frac{p(x)}{q(x)}+\frac{e / j}{\left((x-\gamma)^{2}+1 / j\right) q(x)} ;
$$

then $R\left(A^{j}, \cdot\right) \in R_{m}^{n}[\alpha, \beta]$. We have $R\left(A^{j}, \gamma\right)=p(\gamma) / q(\gamma)+e=f(\gamma)$ and $\left\{R\left(A^{j}, \cdot\right)\right\} \rightarrow R\left(A^{*}, \cdot\right)$ uniformly on $X_{k} \sim\{\gamma\}$; hence $\left\|f-R\left(A^{j}, \cdot\right)\right\|_{k} \rightarrow e$ $-1 / k$. Let $R\left(A^{*}, \cdot\right)$ have degree $l$; then there exists $\left\{x_{0}^{k}, \ldots, x_{l}^{k}\right\} \in X_{k}$, where $\alpha \leqslant x_{0}^{k}<\cdots<x_{l}^{k} \leqslant \beta$ and $\gamma$ is one of the set, such that $f-R\left(A^{*}, \cdot\right)$ alternates in sign on the set and has the absolute value $e-1 / k$ except on $\gamma$. By arguments similar to those of Theorem 1, no best approximation to $f$ exists on $X_{k}$.

Theorem 3 could be generalized by defining a type of irregularity at an interior point, namely taking an arbitrary value on the point and being uniformly close except on a neighbourhood of that point. However, there may exist no other family of interest for which such a type of irregularity holds.

\section{REFERENCES}

1. R. B. Barrar and H. L. Loeb, On N-parameter and unisolvent families, J. Approximation Theory 1 (1968), 180-181. MR 38 \#4873.

2. C. B. Dunham, Chebyshev approximation with respect to a weight function, J. Approximation Theory 2 (1969), 223-232. MR 40 \#6137.

3. - Chebyshev approximation by $A+B^{*} \log (1+C X)$. II, J. Inst. Math. Appl. 10 (1972), 369-372.

4. - Approximation by alternating families on subsets, Computing 9 (1972), 261-265. MR 47 \# 1242.

5. - Approximation by exponential-polynomial products on finite point sets, J. Inst. Math. Appl. 10 (1972), 125-127.

6. Alternating Chebyshev approximation, Trans. Amer. Math. Soc. 178 (1973), 95-109. MR 47 \# 7282.

7. - Varisolvent Chebyshev approximation on subsets, Approximation Theory, Academic Press, New York, 1973, pp. 337-340. MR 48 \#11859.

8. - Nonexistence of best rational approximations on subsets, J. Approximation Theory 14 (1975), 160-161.

9. - Chebyshev approximation by logarithmic families, Z. Angew. Math. Mech. 53 (1973), $352-353$.

10. J. R. Rice, The approximation of functions, Vol. 2, Addison-Wesley, Reading, Mass., 1969. MR 39 \# 5989.

Department of Computer Science, University of Western Ontario, London, Ontario, CANADA 